\title{
PARASITÓIDES ASSOCIADOS A CRYPTOBLABES GNIDIELLA (LEPIDOPTERA, PYRALIDAE) EM VIDEIRA, RS
}

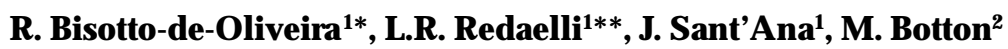

IUniversidade Federal do Rio Grande do Sul, Faculdade de Agronomia, Departamento de Fitossanidade, Laboratório de Biologia, Ecologia e Controle Biológico de Insetos, Av. Bento Gonçalves, 7712, CEP 91540-000. Porto Alegre, RS, Brasil. E-mail: ricbisotto@yahoo.com.br

RESUMO

\begin{abstract}
A traça-dos-cachos Cryptoblabes gnidiella tem se destacado como praga importante no cultivo davideira em Bento Gonçalves, RS, Brasil. Como objetivo deverificar aincidência deparasitismo sobreas formas imaturas deC. gnidiella foramreal izadasamostragensquinzenais decachoseramos em 2 pomares deV itis vinifera, mantidos com esem aplicação deinsetici das, no período dejulho/ 2004ajulho/ 2005. O material coletado foi examinado em laboratório com auxílio demi croscópio estereoscópio Wild M5separando-seaté100 lagartas e 100 pupas deC. gnidiella decada pomar por amostragem. Osimaturos foram manti dos em câmara climatizada com temperatura constantede 25ㅇ $\mathrm{C}$ até a emergência dos adultos da traça ou de parasitóides. Desse material registrou-se a emergência dos Hymenoptera A panteles sp. (Braconidae), Perilampidae, Pimpla croceiventris (Cresson) (Ichneumonidae), V enturia sp. (Ichneumonidae) eM acrocentrus sp. (Braconidae).V enturia sp. foi o parasitói demaisabundantenos 2 pomares. Foi constatada umamaior riqueza deespécies no pomar sem aplicação de inseticidas.
\end{abstract}

PALAVRAS-CHAVE: Cryptoblabes, traça-dos-cachos, parasitismo, videira.

\section{ABSTRACT}

PARASITOIDS ASSOCIATED WITH CRYPTOBLABES GNIDIELLA (LEPIDOPTERA, PYRALIDAE) IN GRA PEVINE, STATE OF RIO GRANDE DO SUL, BRAZIL. Thehoneydew moth, Cryptoblabes. gnidiella, is an important peston grapevineorchards, in Bento Gonçalves, stateof Rio Grande do Sul, Brazil. This study was aimed to verify the parasitism on immatures stages of $C$. gnidiella. Samples of grapes and branches in two orchards of Vitis vinifera, managed with and without insecticidetreatment, werecarried outfromJuly/ 2004toJuly/2005. Biweekly observations reveal ed, at most, 100 larvae and 100 pupae of $C$. gnidiella from each orchard area. The immature stageswerekeptin an accl imatized chamber $\left(25 \pm 1^{\circ} \mathrm{O}\right.$ ) until theemergenceof adultsor parasitoids. Five species of Hymenoptera were found associated with C. gnidiella: A panteles sp. (Braconidae), Perilampidae, Pimpla croceiventris (Cresson) (Ichneumonidae), V enturia sp. (Ichneumonidae) and $M$ acrocentrus sp. (Braconidae).V enturia sp. was the most abundant parasitoidin thetwo orchards. More species were observed in the orchard without application of insecticides.

KEY WORDS: Cryptoblabes, honeydew moth, parasitism, vineyards.

\section{INTRODUÇÃO}

A traça-dos-cachos, Cryptoblabes gnidiellaMillière (Lepidoptera, Pyralidae), nativada região Mediterrânea, temsidoconsideradaumadasprincipaispragas da videira, no Rio Grande do Sul (RINGEnBerg et al., 2006). A s lagartas al imentam-se dos cachos de uva, ondeprovocamoapodrecimentoeaquedadasbagas, podendo acarretar também o desenvolvimento e a proliferação de fungos e bactérias causadores de podridões na pré-colheita (RINGENBERG et al., 2006).

A espécietambéméregistrada naÁ frica, A mérica do Sul, Ásia eEuropa (BA GNoll; LucCHI, 2001), associadaaumaamplavariedadedehospedeiros (SWAILEM; ISMAIL, 1972; SCATONI; BentanCOURT, 1983; YehUda etal., 1991; Yenuda et al., 1992; Molina, 1998).

\footnotetext{
Embrapa Uva e Vinho, Bento Gonçalves, RS, Brasil.

*Programa de Pós-Graduação em Fitotecnia - Bolsista CA PES.

**Bolsista CNPq.
} 
Os danos provocados pela traça-dos-cachos têm sido minimizados através da utilização de inseticidas (ISHAAYA et al ., 1983; SINGH; SINGH, 1998) que, embora evitem perdas decorrentes da traça, podem acarretar prejuízosambientais como adiminuição daabundância e diversidade de inimigos naturais no agroecossistema.

São poucosos registros deespécies deparasitóides associados a C. gnidiella. No Egito, em laboratório, SWAILEM; IsmaIL (1972) registraram Phanerotoma sp. (Hymenoptera, Braconidae) provenientedepupasde C. gnidiella eumasegundaespéciedeBraconidae. Em Israel, na cultura do abacate, o parasitóide de ovos, provenientedaCalifórnia(EUA), T richogrammaplatneri Nagarkatti (Hymenoptera, Trichogrammatidae) foi introduzido paracontrolar atraça-dos-cachos; entretanto, os autoresnão referemsehouveefetivo controle eestabel ecimento da espécie (WYSOKI; RENNEH, 1985). Na Itália, BA GN OLI;L UCCHI (2001) registraramemvideira, lagartas de C. gnidiella parasitadas pelos himenópteros Itoplectis sp. (Ichneumonidae, Pimplinae) eP haner otomasp. (Braconidae, Cheloninae). BENTANCOURT; SCATONI (2001), noUruguai, sereferiram a A panteles desantisi (Blanchard) (Hymenoptera, Braconidae) como parasitóide deC. gnidiella.

Além da importância que os himenópteros parasitóidestêm parao controlebiológico deespécies consideradas pragas, estes têm sido largamenteutilizados como indicadores biológicos em inventariamentos deecossistemas por apresentarem uma grande riqueza de espécies e diversidade de táxons, quesão afetadospor modificaçõesno ambiente(LeWIS; WhitfieLD, 1999). No Brasil, eespecialmente no Rio Grande do Sul, não existem trabal hos que abordamo parasitismo sobreatraça-dos-cachos. Este estudo visou registrar a ocorrência, a riqueza, a eqüitabilidade e a dominância de parasitóides em populações deimaturos deC. gnidiella emdoispomares deV . vinifera dacultivar PinotNoir, mantidoscom esemaaplicação deinseticidasemBentoGoçalves, RS.

\section{MATERIAL E MÉTODOS}

O trabal ho foi conduzido em Bento Gonçalves (2910'S5132'O), RS, si tuada na “Encosta Superior do N ordeste do Rio Grandedo Sul" (EMBRAPA, 2006). O clima daregião édo tipo temperado úmido segundo a classificação climática de Köppen (MoRENO, 1961). Dois pomares de Vitis vinifera da CV. Pinot Noir com 0,16 ha cada e 4 anos de idade, instalados no sistema deespal deira emantidossob manejo convencional com histórico de ataque da traça-dos-cachos foram escol hidos para realizar o levantamento. Um dos pomares ( $T$ ) foi mantido no sistema convencional de manejo, incluindo 3 apli- cações de inseticidas real izadas em 16/ 9 e 17/ 11/ 2004 , com o inseticida fenitrotiona ( $150 \mathrm{~mL} / 100 \mathrm{~L}$ ) e em 5/ 1/ 2005 com espinosade ( $20 \mathrm{~g} / 100 \mathrm{~L}$ ). 0 pomar não tratado (NT) recebeu o mesmo manejo exceto as aplicações de inseticidas. Os pomares estavam distanciados entre si por aproximadamente $1.000 \mathrm{~m}$. A face sul do pomar T fazia divisa coma RS 444, a nortecom outro pomar deV . vinifera cv. Pinot $\mathrm{N}$ oir, a leste com um pomar deV . vinifera cv. Merlot ea oeste com um pomar deV . labrusca cv. Bordô. A face sul do pomar NT fazia divisa com uma estrada secundária, a nortecom um pomar de V . Iabrusca cv. Isabel, a leste com uma área de vegetação arbustiva decrescimento espontâneo ea oeste com um pomar deV. vinifera cv. Merlot.

Cada pomar foi subdividido em 4 subáreas de igual tamanho para assegurar a representatividade dasamostragens realizadasquinzenal mente. Emcada ocasião, sempre que presentes, foram coletados e individual izados em sacos plásticos 2 cachos e um ramo de $40 \mathrm{~cm}$ decadaum dos 40 pontos previamente sorteados. Emlaboratório, os cachoseos ramosforam examinados sob microscópio estereoscópio, registrando-se o número de ovos, lagartas e pupas de C. gnidiella. Decadacoleta, separou-se100lagartase 100 pupas ou o maior número de indivíduos encontrado nestes estágios. Para assegurar a representatividade das áreas amostrais, sempre que presentes foram individualizadas 25lagartase25pupas por subárea. Quando não foi possível atingir as 25 unidades em alguma das subáreas, completou-se a quantidade faltantecomindivíduosexcedentes deoutrassubáreas atéolimitede100 lagartas e100 pupas por pomar. As lagartas foram transferidas para tubos de vidro ( 25 $\mathrm{mm} \times 80 \mathrm{~mm}$ ) contendo dieta artificial utilizada para a criação de Argyrotaenia sphaleropa (Meyrick) (Lepidoptera, Tortricidae) (MANFredi-Coimbra et al., 2005). As pupas foram colocadas em recipientes de plástico ( $30 \mathrm{~mm} \times 15 \mathrm{~mm}$ ), contendo al godão umedecido com água destilada e coberto com película de PVC. Os imaturos foram mantidos em câmara climatizada ( $25 \pm 1$ o C; fotofasede 12 h) atéa emergência dos lepidópteros ou de parasitóides.

Os dados foram organizados de acordo com os estágiosfenológicosdavideiradescritosporM MNDELLI et al. (2003). Com base neste autor e considerando o observado ao longo das amostragens, puderam ser reconhecidoscinco períodos dentrodocicl ofenológico dacultura: 1) início dabrotação-6/ 9/ 2004, 2) floração - 4/ 10/ 2004a 31/ 10/ 2004, 3) cachos verdes - 1/ 11/ 2004a23/ 12/2004(início daformação das bagas até o momento em que $50 \%$ das bagas ainda apresentavam coloração verde), 4) cachos maduros - 24/ 12/ 2004 a 20/ 3/ 2005 (início quando mais de 50\% das bagas haviam mudado de coloração e término no momento em que até $50 \%$ das bagas apresentavam 
turgidez) e 5) cachos secos 21/ 3/ 2005 a 11/ 7/ 2005 (período pós-colheita - sendo que os cachos que permaneceram nos pomares estavam com mais de $50 \%$ das bagas murchas ou secas). A poda seca foi realizada em 16/ 8/ 2004.

O número de indivíduos registrado nos dois pomaresenos diferentes estágiosfenológicos dacultura foramcomparadospelotesteKruskall-WalliseM annWhitney.

As comunidades deparasitóides, nos dois pomares, foram descritas pelo número de indivíduos (N) e ariquezadeespécies(S). Foramcal culados, conforme MoReno (2001), osíndices dediversidadedeM argalef, Shannon-WienereSimpson. Osíndicesdediversidade entre os dois pomares foram comparados através do testeB ootstrapping, utilizando-seo programa Past versão 1.34 (H AMm ER et al., 2001). Os parasitóides emergidos foram enviados para especialistas para identificação.

Nosresultadosapresentadosepara os cálculosde percentual deparasitismo foram desconsideradosos insetos que morreram antes de chegar à fase adulta.

\section{RESULTADOSE DISCUSSÃO}

Ao longo do experimento foram registradas 5 espécies de hi menópteros parasitóides associadas aC . gnidiella: Pimpla croceiventris (Cresson, 1868) (Ichneumonidae, Pimplinae), Venturia sp. (Ichneumonidae, Campopleginae), A panteles sp. (Braconidae, Microgastrinae), M acrocentrus sp. (Braconidae, Macrocentrinae) e 1 espécie de Perilampidae (Chalcidoidea).

A panteles sp., M acrocentrus sp. e a espécie de Perilampidaeforam observadas somentesobrelagartas, enquanto queP. croceiventris apenas em pupas e $\checkmark$ enturiasp. em lagartas epupas datraça-dos-cachos.

Estessãoosprimeirosregistros dessesparasitóides associados aC. gnidiella, com exceção deA pantel essp., cuja identificação específica não foi possível no presente estudo, impossibilitando a afirmação de que trata-se de A. desantisi referida por BENTANCOURT; SCATONI (2001), no Uruguai.

O parasitismo em C. gnidiella foi verificado no pomarT apenas no período em queoscachosestavam secos enquanto queno pomar NT, estefoi constatado no período de cachos maduros esecos. Em ambos os pomaresnãoforam registradosimaturosdeC.gnidiella no período de cachos verdes.

Emrelação ao parasitismo, no pomar $T$, no período de cachos secos, de 590 lagartas, 6,4\% (38) estavam parasitadas por $V$ enturia sp. Do total de pupas avaliadas (176), em 13,6\% (24) constatou-se parasitóides, sendo $12,5 \%$ (3) Venturia sp. e $87,5 \%$ (21) de $P$. croceiventris.
No pomar NT, no período de cachos maduros, apenas lagartas (32) foram amostradas; destas 3,1\% (1) estavam parasitadas por A panteles sp. No período de cachos secos, de 673 lagartas, 15\% (101) estavam parasitadas, sendo 3\% (3) porA pantel es sp., 1\%(1) por M acrocentrus sp., 1\% (1) por uma espécie de Perilampidae e 95\% (96) por V enturia sp. Em relação às pupas, de 75 observadas, 25,3\% (19) estavam parasitadas, 47,4\% (9) por $P$, croceiventris e $52,6 \%$ (10) por $V$ enturia sp.

$\checkmark$ enturiasp. foi aespéciedeparasitóidemaisabundante nos dois pomares; entretanto, no pomar NT o número de indivíduos parasitados por esta espécie foi significativamente maior ( $P=0,03)$.

Nos 2 pomares (TeNT) os maiores percentuais de parasitismo emC. gnidiella foramverificadosno período de cachos secos. Estes resultados sugerem uma preferênciatantodofitófago, quantodosparasitóides, por este período, em função de condições da própria cultura ou de fatores abióticos, como temperatura e umidade relativa, prevalentes nesta época.

Com o início do processo de desidratação dos cachos ocorre a concentração de açúcares nas bagas, proporcionando mel hores recursos al imentares tanto para os adultos quanto paraas lagartas da traça-dos-cachos (SCATONI; BENTAN COURT, 1983; SILva; Mexia, 1999; Bagnoli; Lucchi, 2001). Swailem; ISMAIL (1972) registraram quea ocorrência da traçados-cachos está freqüentemente associada à presençadepulgõesecochonilhas em função dosseus exsudatos, o mesmo sendo constatado por SILVA; MeXIA (1999).

EmToscana, Itália, BA GN OLI; LuCCHI (2001)registraramamai or quantidadedeadultosemarmadilhas de feromônio no período em que os cachos estavam em adiantado estágio de maturação e/ ou apodrecimento, evidenciando que a fenologia e os voláteis das plantas estariam atrai ndo os adultos da traça.

Segundo SANT'ANA; CorrêA (2001), substâncias secundárias liberadas pelas plantas foram detectadas por herbívoros queas usavam como cairomônio, podendo estes, atuar como pistas químicas tanto no comportamento reprodutivo, como na localização e seleção do hospedeiro.

A liberação de voláteis pela planta e/ ou pelo processo de fermentação das bagas poderia estar sinalizando sítios de acasalamento, oviposição e a disponibilidade de recursos al imentares, tanto para os adultos da traça, quanto para os parasitóides.

Osexcrementos demuitosinsetostambémemitem odoresaosquaisosparasitóidesrespondem positivamente. Bentan COURT; SCATONI (2001) sugeriram que fêmeas de Habrobracon herbetor (Say) (Hymenoptera, Braconidae) e Venturia canescens (Gravenhorst) (Hymenoptera, Ichneumonidae) são atraídas por voláteis desta natureza. 
Tabela 1- N úmero deindivíduos( $\mathrm{N}$ ), número deespécies (S) e índices de Margalef $\left(D_{M q}\right)$, Shannon-Wiener $\left(H^{\prime}\right)$ e Simpson $(\lambda)$ dos parasitóides registradosemimaturos de $C$. gnidiella, no período de cachos secos, nos pomares de $\mathrm{V}$ itis vinifera cv. Pinot N oir, com aplicação de inseticidas (T) e sem inseticidas (NT). Bento Gonçalves, RS. (julho/ 2004 a julho/ 2005).

\begin{tabular}{lll}
\hline & \multicolumn{2}{c}{ Pomares } \\
\cline { 2 - 3 } & $\mathrm{T}$ & $\mathrm{NT}$ \\
\hline $\mathrm{N}$ & 62 & 120 \\
$\mathrm{~S}$ & 2 & 5 \\
$\mathrm{D}_{\mathrm{Mg}}$ & 0,2423 & 0,8355 \\
$\mathrm{H}^{\prime}$ & 0,6402 & 0,4759 \\
$\lambda$ & 0,4480 & 0,2133 \\
\hline
\end{tabular}

Os percentuais de parasitismo, no período de cachos secos, tanto em lagartas $(P=0,09)$ quanto em pupas $(P=0,38)$ de $C$. gnidiella não diferiram significativamente entre os pomares quando comparados pelo teste de Bootstraping.

Comparando-se os pomares em relação à riqueza de espécies de parasitóides, verificou-se que a do pomar NT foi significativamente maior do quea do T ( $P$ =0,032). Da mesma forma, o índice de Simpson ( $)$ apontou queo pomar NT apresentadiversidadesignificativamentemaior $(P=0,005)$ do queo pomar T. Já o índice de Shannon-Wiener $\left(\mathrm{H}^{\prime}\right)$ não apontou diferençassignificativasnadiversidadedeparasitóidesentre os 2 pomares ( $P=0,224)$ (Tabela 1 ). A maior abundância e dominância de Venturia sp. no pomar NT e a equitabilidade das duas espécies encontradas no pomar T são os fatores que podem melhor explicar as diferenças encontradas nos índices de diversidade.

Um dos fatores que podeter influenciando a diferença de diversidade, com relação ao parasitismo observado nos dois pomares, foi o manejo utilizado. O emprego deinseticidas, apesar denão ter afetado a população de $C$. gnidiella por ter sido aplicado em períodosem quea espécieainda não havia seestabelecido no campo, podeter diminuídoa populaçãodos parasitóides. N ão existem informações a respeito da seletividade dos inseticidas utilizados sobre inimigos naturais da traça-dos-cachos, send o desconhecido o impacto destes produtos, a longo prazo, sobre essas populações.

Outrofator quepodeter beneficiandoaocorrência de parasitóides no pomar NT está relacionado à presença de uma área com vegetação arbustiva de crescimento espontâneo, situada contígua à sua face leste. Essa vegetação composta por espécies diversas, poderia estar servindo de refúgio aos inimigos naturais, fornecendo alimento ou mesmo abrigando outros hospedeiros das espécies de parasitóides registradas.
O entendimento acerca da diversidade, da estrutura edacomposição da comunidadedeparasitóides, bem como da ação destes sobre populações de $C$. gnidiella poderá fornecer importantes informações para o manejo e controle desta espécie.

\section{AGRADECIMENTOS}

Os autores agradecem a identificação dos parasitóides, efetuadapelaDra.TâniaM araGuerra, da Universidade Federal de Santa Catarina e pelo Dr. Juan José Martinez, da Divisão de Entomologia do Museu Argentino de Ciências $\mathrm{N}$ aturais "Bernardino Rivadavia", La Plata, Argentina. A Vinícola Miolo pela disponibilização do pomar, ao Consel ho $\mathrm{N}$ acional dePesquisa eDesenvolvimento Científico e Tecnológico (CN Pq) pela concessão de Bolsa de Produtividade para segundo autor e a Coordenadoria de A perfeiçoamento de Pessoal de Nível Superior (CAPES) pela concessão de bolsa para o primeiro autor.

\section{REFERÊNCIAS}

BAGNOLI, B.; LuCCHI, A. Bionomics of Cryptoblabes gnidiella (Millière) (Pyralidae, Phycitinae) in Tuscan vineyards. IOBC wprs Bulletin, v.24, n.7, p.79-83. 2001.

Bentancourt, C.M.;Scatoni, I.S. Enemigos naturales, manual ilustrado para la agricultura y la forestación. Montevideo: Facultad deAgronomia, PREDEG/ GTZ, 2001. 169 p.

EMBRAPA. Centro Nacional dePesquisadeUvaeVinho. Dadosmeteorológicosmensaisfornecidos pela estação agroclimática - Bento Gonçalves-RS. Disponível em: <http:/ / www.cnpuv.embrapa.br/ meteorologia/ bento- mensais.html >. A cesso em: 24 jan. 2006.

Hammer, O.; Harper, D.A.T.; RIAn, P.D. Past: Palaeonthological statistics software package for education and data analysis. Pal aentologia Electronica, v.4, n.1, p.1-9, 2001. Version. 1.37. Disponível em: \http:/ / palaeo-electronica.org/ 2001 1/ past/ issuel 01.htm>. Acesso em: 12 nov. 2005.

ISHAAYA, I.; G UREVITZ, E.; A SCHER, K.R.S. Syntheticpyrethroids and avermectin for controlling pests Lobesia botrana, Cryptoblabes gnidiella and D rosophila melanogaster. Phytoparasitica, v.11, n.3/ 4, p.161-166, 1983.

LeWIS, C.; WhITfIELD, J.B. Braconidaewasp (Hymenoptera: Braconidae) diversity in forest plots under different silvicultural methods. Environmental Entomology,v.28, p.986-997, 1999.

Mandelli, F.; Berlato, M. A.; Tonietto, J.; Bergamaschi, H. Fenologia da videira na Serra Gaúcha. Pesquisa A gropecuaria Gaúcha, v.9, n.1/ 2, p.129-144, 2003.

Manfredi-Colmbra, S.; Garcia, M.S.; L oeck, A.E.; Botton , M.; Forestı, J. A spectos biológicos de Argyrotaenia sphal eropa (Meyrick, 1909) (Lepidoptera: Tortricidae) em dietas artificiais com diferentes fontes protéi cas. Ciência Rural, v.35, n.2, p.259-265, 2005. 
Molina, J.M. Lepidopteros associados al cultivo del arándano en A ndalúcia Occidental. Boletin deSanidad V egetal Plagas, v.24, n.4, p.763-772, 1998.

Moreno, C.E. M étodos para medir la biodiversidad. Zaragoza: CYTED, 2001. 84p.

Moreno, J.A. Clima do Rio Grande do Sul. Porto Alegre: Secretaria da A gricultura, 1961. 42p.

PARÉ,P.W.;T UMLINSON, J.H.Plantvolatilesignalsin response to herbivore feeding. In: BEHAVIORAL ECOLOGY SYMPOSIUM , 2., 1996, Florida. A nais. Florida, 1996. p.93-103.

Ringenberg, R.;Botton ,M.;Garcia, M.S.;A morim,F.M.; HaJI, F.N. A traça-dos-cachos da videira. Revista Cultivar HF , n.35, p.31-33, 2006.

SANT'ANA, J.; CORRÊA, A.G. Fundamentos da comunicação químicadeinsetos. In:FerreIRA, J.A.C.;V IEIRA, P. (Org.). Produtos naturais no controle de insetos. São Carlos: UFSCar, 2001. p.9-22.

Scatonı, I.B.; Bentancourt, C.M. Cryptoblabes gnidiella (Millière): una nueva lagarta de los racimos en los viñedos de nuestro pais. Revista de la AIA, v.1, n.4, p.266-268, 1983.

Silva,E.B.; M exIA, A. Thepest complexCryptoblabesgnidiella (Millière) (Lepidoptera: Pyralidae) and Planococcus citri (Risso) (H omoptera: Pseudococcidae) on sweet orange groves (Citrus sinensis (L.) Osbeck) in Portugal: interspecific association. Boletin Sanidad V egetal, n.25, p.89-98, 1999.

SINGH, Y.P.; SINGH, D.K. Host plants, extent of damageand seasonal abundance of earhead caterpillar, Cryptoblabes gnidiella Miller. A dvances in A gricultural Research in India, v.7, p.133-137, 1997.

SwaILem, S.M.; IsmaIL, I.I. On the biology of the honey dew moth Cryptoblabes gnidiella, Millière. Bulletin dela Societe Entomologique d'Egypte, v.56, p.127-134, 1972.

WYSOKI, M.; RENNEH, S. Introduction into Israel of Trichogramma platneri N agarkatti, an egg parasiteof lepidoptera. Phytoparasitica, v.13, n.2, p.139-140, 1985.

YehudA, S.B.; Wrsokı, M.; Rosen, D. Phenology of the honeydew moth, Cryptoblabes gnidiella (Millière) (Lepidoptera: Pyralidae), on avocado. Journal of Entomology, v.25/ 26, p.149-160, 1991-1992.

Recebido em 2/ 8/ 06 Aceito em 10/ 4/ 07 